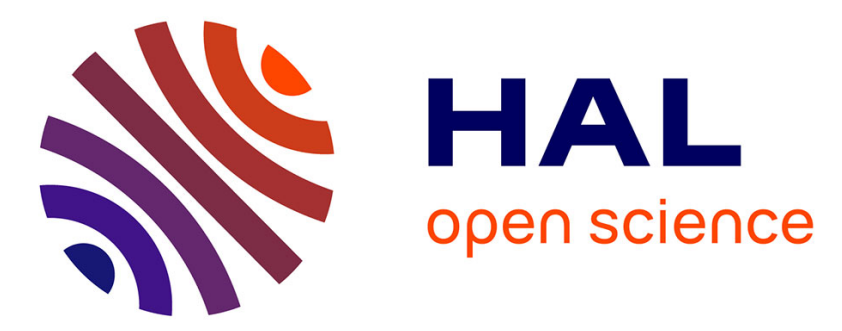

\title{
Idealized Algol with ground recursion, and PDA equivalence
}

\author{
Andrzej Murawski, Luke Ong, Igor Walukiewicz
}

\section{To cite this version:}

Andrzej Murawski, Luke Ong, Igor Walukiewicz. Idealized Algol with ground recursion, and PDA equivalence. ICALP'05, 2005, Portugal. pp.917-926. hal-00335728

\section{HAL Id: hal-00335728 \\ https://hal.science/hal-00335728}

Submitted on 30 Oct 2008

HAL is a multi-disciplinary open access archive for the deposit and dissemination of scientific research documents, whether they are published or not. The documents may come from teaching and research institutions in France or abroad, or from public or private research centers.
L'archive ouverte pluridisciplinaire HAL, est destinée au dépôt et à la diffusion de documents scientifiques de niveau recherche, publiés ou non, émanant des établissements d'enseignement et de recherche français ou étrangers, des laboratoires publics ou privés. 


\title{
Idealized Algol with Ground Recursion, and DPDA Equivalence ${ }^{\star}$
}

\author{
A. S. Murawski ${ }^{1}$ \\ C.-H. L. Ong ${ }^{1}$ \\ I. Walukiewicz ${ }^{2}$ \\ 1 Oxford University Computing Laboratory, Parks Road, Oxford OX1 3QD, UK \\ 2 LaBRI, Université Bordeaux-1, 351, Cours de la Libération, 33 405, Talence, France
}

\begin{abstract}
We prove that observational equivalence of $\mathrm{IA}_{3}+\mathbf{Y}_{0}$ (3rdorder Idealized Algol with 0th-order recursion) is equivalent to the DPDA Equivalence Problem, and hence decidable. This completes the classification of decidable fragments of Idealized Algol. We also prove that observational approximation of $\mathrm{IA}_{1}+\mathbf{Y}_{0}$ is undecidable by reducing the DPDA Containment Problem to it.
\end{abstract}

\section{Introduction}

Observational equivalence is an extensional notion of program equivalence. Two program phrases are observationally equivalent if one can be replaced by the other in any program without causing any observable difference to the computational outcome. Reynolds's Idealized Algol (IA) is an elegant and compact programming language that combines imperative programming with high-order features, mediated by a simple type theory. Observational equivalence in IA is in general undecidable even when ground types are finite sets. This paper is concerned with the question of decidability of observational equivalence for appropriate fragments of IA.

We begin with a quick review of IA. Ground types of IA, which are ranged over by $\beta$, are exp (expressions), com (commands) and var (assignable variables). Types of IA, ranged over by $\theta, \theta^{\prime}$ etc., are generated from ground types by the function space constructor $\theta \rightarrow \theta^{\prime}$. The order of a type is defined by $\operatorname{ord}(\beta)=0$ and $\operatorname{ord}\left(\theta \rightarrow \theta^{\prime}\right)=\max \left(\operatorname{ord}(\theta)+1\right.$, ord $\left.\left(\theta^{\prime}\right)\right)$. Finitary Idealized Algol, IA $\mathrm{f}$, is just recursion-free Idealized Algol over finite ground types. An $\mathrm{IA}_{\mathrm{f}}$-term, $\Gamma \vdash M: \theta$, is an ith-order term provided the types of the free identifiers in $\Gamma$ are of order less than $i$ and $\operatorname{ord}(\theta) \leq i$. The collection of $i$ th-order IA $_{\mathrm{f}}$ terms will be denoted by $\mathrm{IA}_{i}$. We can extend $\mathrm{IA}_{\mathrm{f}}$ with iteration by adding the rule

$$
\frac{\Gamma \vdash M: \exp \quad \Gamma \vdash N: \text { com }}{\Gamma \vdash \text { while } M \text { do } N: \operatorname{com}}
$$

and with general recursion by adding the rule

$$
\frac{\Gamma, x: \theta \vdash M: \theta}{\Gamma \vdash \mu x^{\theta} \cdot M: \theta} .
$$

\footnotetext{
* Work supported by the UK EPSRC (GR/R88861/01), the European Research Training Network GAMES and St John's College, Oxford.
}

reference to Figure 2 removed 
We write $\mathrm{IA}_{i}+\mathbf{Y}_{j}$ to mean $\mathrm{IA}_{i}$ augmented by the collection of $i$ th-order terms of the shape $\Gamma \vdash \mu x^{\theta} . M: \theta$ where $\operatorname{ord}(\theta) \leq j$. We tabulate all known results on the complexity of observational equivalence of $\beta$-normal terms in IA [1-4]:

\begin{tabular}{c|c|c|c|c} 
& pure & + while & $+\mathbf{Y}_{0}$ & $+\mathbf{Y}_{1}$ \\
\hline \hline $\mathrm{IA}_{1}$ & CONP & PSPACE & $?$ & undecidable \\
$\mathrm{IA}_{2}$ & PSPACE & PSPACE & $?$ & undecidable \\
$\mathrm{IA}_{3}$ & ExPTIME & ExPTIME & $?$ & undecidable \\
$\mathrm{IA}_{4}$ & undecidable & undecidable & undecidable & undecidable
\end{tabular}

The same results as above also hold for observational approximation.

This paper addresses the cases marked with question marks. In $\mathrm{IA}_{i}+\mathbf{Y}_{0}$ only programs of ground type can call themselves recursively. For example, whileloops while $b$ do $c$ can then be defined by

$$
b: \exp , c: \operatorname{com}, z \vdash \mu z^{c o m} \text {. ifzero } b \operatorname{skip}(c ; z) .
$$

We show that observational equivalence in $\mathrm{IA}_{i}+\mathbf{Y}_{0}$ is decidable for $i=1,2,3$ by giving a reduction to the DPDA Equivalence Problem (recently proved decidable by Sénizergues [8]). This does not tell much about the complexity, though. At the moment it is only known that the complexity of DPDA Equivalence is bounded by a primitive recursive function [5]. We also show that already for $i=1$ observational equivalence is at least as hard as DPDA Equivalence. In consequence, no advance on the complexity of the former problem can be made without an advance on the latter. Another result is that observational approximation in $\mathrm{IA}_{i}+\mathbf{Y}_{\mathbf{0}}$ is undecidable for $i=1,2,3$, because the undecidable DPDA Containment Problem [6] can be reduced to it.

Let us comment on the relationship of our results to a recent paper [4] showing decidability of $\mathrm{IA}_{3}+$ while. In that paper a simpler language was considered but it was translated to a weaker form of pushdown automata. This was essential to get a precise complexity bound. In this paper we model a richer language but do not concentrate on complexity, hence our constructions are not designed to optimize the size of the resulting automata. In [4] most constructions relied on parallel composition of automata. This is not possible here as DPDAs are not closed under that operation.

\section{Game semantics: complete plays}

We assume familiarity with the treatment of game semantics of IA as presented, for example, in [7]. Recall that the multiplicative composition $\sigma ;_{\mathrm{m}} \tau: A \multimap C$ of two strategies $\sigma: A \multimap B$ and $\tau: B \multimap C$ is defined by parallel composition with hiding by letting the strategies interact in the shared subgame $B$ and subsequently hiding the $B$-moves.

Let $\sigma: A \Rightarrow B$ and $\tau: B \Rightarrow C$. Recall that $A \Rightarrow B=! A \multimap B$ and $B \Rightarrow C=$ $! B \multimap C$. In order to compose the strategies, one first defines $\sigma^{\dagger}: ! A \multimap ! B$ by

$$
\sigma^{\dagger}=\left\{s \in L_{! A-! ! B} \mid \text { for all initial } m, s\lceil m \in \sigma\},\right.
$$


where $s \uparrow m$ stands for the subsequence of $s$ (pointers included) whose moves are hereditarily justified by $m$. Then $\sigma ; \tau: A \Rightarrow C$ is taken to be $\sigma^{\dagger}$; $\tau$.

Given a set $\sigma$ of positions on $G$ we write $\mathcal{L}(\sigma)$ for the set of the underlying sequences of moves from $M_{G}$. For a given strategy $\sigma$, we write comp $\sigma$ for the set of its non-empty plays in which the number of questions matches the number of answers; such plays are called complete.

Remark 1. We will be interested in the $\dagger$ construction when $B=\llbracket \theta \rrbracket$ and $\operatorname{ord}(\theta) \leq 1$. Then $\sigma^{\dagger}$ can be characterized explicitly as follows.

$-B=\llbracket \beta \rrbracket$ : Then $\sigma^{\dagger}=(\operatorname{comp} \sigma)^{*} \sigma$, i.e. $\sigma^{\dagger}$ simply iterates $\sigma$.

$-B=\llbracket \beta_{1} \rightarrow \beta_{0} \rrbracket:$ Then the switching conditions in the game $! A \multimap ! B$ imply that a new copy of $\sigma$ can be started each time $\sigma$ is finished (as above) and, additionally, after each question $q_{1}$ from $\beta_{1}$. We can thus capture $K=$ $\{\epsilon\} \cup \operatorname{comp}\left(\sigma^{\dagger}\right)$ by the equation below.

$$
K=\{\varepsilon\} \cup \bigcup\left\{q_{0} U q_{1} K a_{1} U \cdots q_{1} K a_{1} U a_{0} K \mid q_{0} U q_{1} a_{1} U \cdots q_{1} a_{1} U a_{0} \in \operatorname{comp} \sigma\right\}
$$

where $U$ 's stand for (possibly empty and possibly different) segments of moves from $A$.

The operational semantics of IA can be found in [7]. We write $M \Downarrow$ if the closed term $M$ reduces to skip. Recall that two terms $\Gamma \vdash M_{1}, M_{2}: \theta$ are observationally equivalent, written $\Gamma \vdash M_{1} \cong M_{2}$, if for any context $C[-]$ such that $C\left[M_{1}\right]$ and $C\left[M_{2}\right]$ are closed terms of type com, we have $C\left[M_{1}\right] \Downarrow$ iff $C\left[M_{2}\right] \Downarrow$. Similarly, $M_{1}$

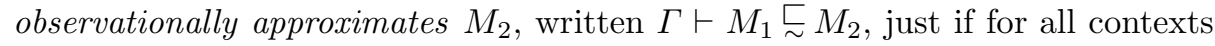
satisfying the properties above, $C\left[M_{1}\right] \Downarrow$ implies $C\left[M_{2}\right] \Downarrow$.

Theorem 1 ([7]). $\Gamma \vdash M_{1} \sqsubseteq M_{2}$ iff comp $\llbracket \Gamma \vdash M_{1} \rrbracket \subseteq$ comp $\llbracket \Gamma \vdash M_{2} \rrbracket$. Consequently, $\Gamma \vdash M_{1} \cong M_{2}$ iff comp $\llbracket \Gamma \vdash M_{1} \rrbracket=\operatorname{comp} \llbracket \Gamma \vdash M_{2} \rrbracket$.

\section{Simple terms and pointer-free representation}

Suppose $\theta=\theta_{1} \rightarrow \cdots \rightarrow \theta_{n} \rightarrow \beta$. Then we write $\operatorname{tail}(\theta)=\beta$. Given $\Gamma \vdash M: \theta$, depending on whether $\operatorname{tail}(\beta)$ is $\operatorname{com}$, exp or var respectively, we define the sets of sequences of moves $(\Gamma \vdash M: \theta)$ by the following decompositions:

$$
\begin{aligned}
\mathcal{L}(\text { comp } \llbracket \Gamma \vdash M: \theta \rrbracket) & =\text { run } \cdot(\Gamma \vdash M) \cdot \text { done } \\
\mathcal{L}(\operatorname{comp} \llbracket \Gamma \vdash M: \theta \rrbracket) & =q \cdot \sum_{j=0}^{\max }\left((\Gamma \vdash M)_{j} \cdot j\right) \\
\mathcal{L}(\operatorname{comp} \llbracket \Gamma \vdash M: \theta \rrbracket) & =\sum_{j=0}^{\max } \operatorname{write}(j) \cdot(\Gamma \vdash M)_{j}^{w} \cdot \text { ok }+ \text { read } \cdot \sum_{j=0}^{\max }\left((\Gamma \vdash M)_{j}^{r} \cdot j\right) .
\end{aligned}
$$

It will turn out convenient to define automata accepting $(\cdots)$ instead of $\mathcal{L}($ comp $\llbracket \cdots \rrbracket)$, because then it will be not necessary to interpret hiding in many 
cases (an operation under which DCFLs are not closed in general). Since $(\cdots)$ are sets of sequences of moves, they do not always represent comp $\llbracket \cdots \rrbracket$ faithfully because they ignore pointers. Nevertheless, we are going to identify a sufficiently rich class of terms for which comp $\llbracket \cdots \rrbracket$ can be recovered from $(\cdots \cdot)$.

First, we note that to establish decidability it suffices to consider $\beta$-normal terms only. This does not solve the pointer problem though. To address it we replace the application rule with its multiplicative version (left) and contraction (right):

$$
\frac{\Gamma \vdash M: \theta \rightarrow \theta^{\prime} \quad \Delta \vdash N: \theta}{\Gamma, \Delta \vdash M N: \theta^{\prime}} \quad \frac{\Gamma, x_{1}: \theta, x_{2}: \theta \vdash M: \theta^{\prime}}{\Gamma, x: \theta \vdash M\left[x / x_{1}, x / x_{2}\right]: \theta^{\prime}} .
$$

All $\beta$-normal terms in $\mathrm{IA}_{3}+\mathbf{Y}_{0}$ are typable if we allow the above rules for $\operatorname{ord}(\theta) \leq$ 2 . We will call a $\beta$-normal $I \mathrm{~A}_{3}+\mathbf{Y}_{0}$ term simple if it can be typed by using the contraction rule only for $\theta$ such that $\operatorname{ord}(\theta)<2$. For instance, $\lambda f . f(\lambda x . f(\lambda y . x))$ is not simple. Note that pointer reconstruction is uniquely defined for all moves except third-order questions (pointers for answers can be reconstructed uniquely thanks to the bracketing condition; first-order moves must point to the unique initial move; finally, because of Visibility, second-order questions must point to the necessarily unique first-order question in the appropriate O-view). As made precise in the lemma below, for simple terms, we can still recover comp $\llbracket \cdots \rrbracket$ from $(\cdots)$.

Lemma 1. Suppose $\Gamma \vdash M: \theta$ is simple. If $s q_{3} \in \llbracket \Gamma \vdash M: \theta \rrbracket$, and $q_{3}$ is a third-order question then $q_{3}$ 's justifier in $s q_{3}$ is the last unanswered enabler of $q_{3}$ in $s$.

Proof. By induction on the structure of simple terms. The crucial point is that if $\sigma: A \multimap B$ satisfies the Lemma for $B=\llbracket \theta \rrbracket$ such that $\operatorname{ord}(\theta) \leq 1$, so does $\sigma^{\dagger}$. This follows from the description in Remark 1.

On the other hand, simple terms are good representatives of $\beta$-normal terms. Any $\beta$-normal term of $\mathrm{IA}_{3}+\mathbf{Y}_{0}$ can be typed by extending a typing derivation of a simple term with a number of applications of the contraction rule for $\theta$ of order 2 followed by a number of applications of the $\lambda$-abstraction rule.

\section{$4 \quad G$-automata}

We are going to use a variant of deterministic pushdown automata. Their states will be divided into O-states and $\mathrm{P}$-states and the stack will be modified and inspected only during $\epsilon$-moves.

- In O-states the automaton will only be able to read an O-move from the input without inspecting or changing the stack; after the transition the state will always change to a P-state.

- In P-states the automaton will either: read a single P-move without modifying the stack and move to an O-state, or perform an $\epsilon$-move and go to a P-state having a possibility to modify a stack. 

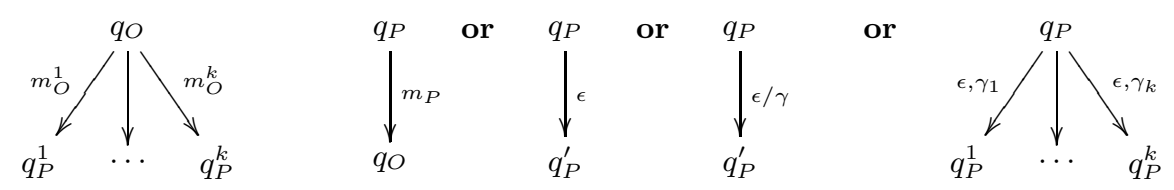

Fig. 1. Transitions in $G$-automata

The constraints are summarized in Figure 1 and captured formally below.

Definition 1. Let $G$ be a game. $A$ G-automaton $\mathcal{A}$ is a tuple $\langle Q, \Omega, i, F, \delta\rangle$ such that

$-Q=Q^{O}+Q^{P}$ is the set of states partitioned into $Q^{O}$ and $Q^{P}$, called the sets of $O$-states and $P$-states respectively;

- $\Omega$ is the stack alphabet;

- $i \in Q^{P}$ is the initial state, $i$ does not have any incoming transitions;

$-F \subseteq Q^{P}$ is the set of final states, final states do not have any outgoing transitions;

$-\delta:\left[Q^{O} \rightarrow\left(M_{G}^{O} \rightarrow Q^{P}\right)\right]+\left[Q^{P} \rightarrow\left(\left(M_{G}^{P} \times Q^{O}\right)+Q^{P}+\left(\Omega \times Q^{P}\right)+\left(\Omega \rightarrow Q^{P}\right)\right)\right]$ is the transition function.

The interpretation of a transition $\delta\left(q_{O}\right) \in M_{G}^{O} \rightarrow Q^{P}$ is that the automaton reads a letter $a \in M_{G}^{O}$ from the input and changes the state to $\delta\left(q_{O}\right)(a)$. Similarly for $\delta\left(q_{P}\right)=\left(b, q_{O}\right) \in M_{G}^{P} \times Q^{O}$, the automaton expects to see $b$ as the input and then changes its state to $q_{O}$. On a transition $\delta\left(q_{P}\right)=q_{P}^{\prime}$ the automaton changes its state but does not consume the input or change the stack. On a transition $\delta\left(q_{P}\right)=\left(\gamma, q_{P}^{\prime}\right)$ the automaton changes the state and pushes $\gamma$ to the stack. On a transition $\delta\left(q_{P}\right) \in \Omega \rightarrow Q^{P}$ the automaton makes a pop move and changes its state depending on the letter that was popped. We will use arrow notation for transitions as in Figure 1. Note that slash and comma denote push and pop operations, respectively.

We write $L(\mathcal{A})$ for the language that $\mathcal{A}$ accepts by final state and the empty stack. It is easy to see that $G$-automata are DPDAs. They have a particularity that they look at the stack only when doing a pop move. We make this restriction to simplify the definitions that follow. It is not difficult to see that for every deterministic pushdown automaton there is an equivalent one with this property.

By a $G$-automaton configuration, we mean a pair $q w$, where $q$ is a state and $w \in \Omega^{*}$ is the content of the stack.

Definition 2. We say that a G-automaton is productive if every non-initial reachable configuration is productive, i.e. the configuration occurs in some accepting run of the automaton.

Lemma 2. For every $G$-automaton there is an equivalent productive automaton. G-added 
$\Gamma$ replaced by $\Omega$ $\pi$ by $\pi^{*}$
Proof. Let $\mathcal{A}=\langle Q, \Omega, i, F, \delta\rangle$ be a $G$-automaton. Consider the function $\beta$ : $\Omega^{*} \rightarrow \mathcal{P}(Q)$ which for all contents of the stack $w \in \Omega^{*}$ returns the set of states $q$ such that the configuration $q w$ is productive. We will first modify $\mathcal{A}$ so that it keeps the current value of $\beta$ in its state. Note that it is easy to update $\beta$ after push-transitions, because $\beta(w a)$ depends only on $\beta(w)$ and $a: q \in \beta(w a)$ iff there exists $q^{\prime}$ such that $q^{\prime} \in \beta(w)$ and when $\mathcal{A}$ starts with $a$ on the stack in state $q$ it can end up in $q^{\prime}$ with empty stack (states $q^{\prime}$ with this property can be precomputed at the very beginning of the construction). In order to handle poptransitions, we simply force the automaton to push the old value of $\beta$ in addition to the pushed stack symbol. Finally, it now suffices to suppress all transitions that are not consistent with $\beta$ to obtain a productive automaton.

Remark 2. (i) The productiveness of the automaton will play an important role in our constructions for several reasons. One is that when $\mathcal{A}$ is productive we can be sure that when running as a subautomaton of some automaton construction (as in the proof of Theorem 2), it will only use the symbols it pushed (it cannot try to make a pop operation on the empty stack as that would be an unproductive configuration). Another consequence of productiveness is that the automaton cannot enter an accepting state while the stack is not empty. Indeed, since there are no outgoing transitions from an accepting state, the resulting configuration would not be productive. Further, as the automaton stack is necessarily empty whenever it reaches a final state, we also know that when it finishes the stack will be exactly as before it has started.

(ii) From an O-state there are in principle several input letters from $M_{G}^{O}$ that can make the automaton advance. However, because of productiveness, in general, not every playable O-move can label an outgoing transition from the O-state. From a P-state there is only one input letter from $M_{G}^{P}$ that the automaton is prepared to read. We have this asymmetry because $G$ automata are designed to accept strategies. Thus, an automaton works in cycles: it reads a letter from $M_{G}^{P}$, then a letter from $M_{G}^{O}$ and then does some internal manipulations on the stack.

Definition 3. We say that an automaton is careful if whenever it reaches a configuration qw after reading the sequence of moves $s$ then the sequence of open second-order questions in $s$ appears in the stack $w$. More formally, there is a function $\pi_{\mathcal{A}}: \Omega \rightarrow M_{G} \cup\{\epsilon\}$ such that $\pi_{\mathcal{A}}^{*}(w)$ is the sequence of open secondorder questions in $s$.

The final definition of this section makes it precise what kind of automata we want to construct. Below, by $\mathcal{A}\left(F^{\prime}\right)$ we will denote the automaton $\mathcal{A}$ with $F^{\prime}$ as the set of accepting states.

Definition 4. We say that a tuple of $G$-automata $\left\langle\mathcal{A}^{1}, \cdots, \mathcal{A}^{n}\right\rangle$, where each $\mathcal{A}^{j}=\left\langle Q^{j}, \Omega^{j}, i^{j}, F^{j}, \delta^{j}\right\rangle$, is fully productive for $\Gamma \vdash M: \theta$ just if each $\mathcal{A}^{j}$ is productive and careful; further

- suppose $\operatorname{tail}(\theta)=$ com: we have $n=1$ and $L\left(\mathcal{A}^{1}\right)=(\Gamma \vdash M: \theta)$ 
- suppose tail $(\theta)=$ exp: we have $n=1, F^{1}=\oplus_{j=0}^{\max } F_{j}$ and $L\left(\mathcal{A}^{1}\left(F_{j}\right)\right)=0 \Gamma \vdash$ $M: \theta)_{j}$ for any $0 \leq j \leq \max$;

- in case tail $(\theta)=$ var: we have $n=\max +2, F^{1}=\oplus_{j=0}^{\max } F_{j}$ and $L\left(\mathcal{A}^{1}\left(F_{j}\right)\right)=$ $(\Gamma \vdash M: \theta)_{j}^{r}$ for any $0 \leq j \leq \max$; for each $0 \leq k \leq \max$, we have $L\left(\mathcal{A}^{k+2}\right)=(\Gamma \vdash M: \theta\rangle_{k}^{w}$.

\section{$5 \quad$ Modelling simple terms}

Here we focus on simple terms. The extension to other $\beta$-normal terms is discussed in Section 6.

Theorem 2. For any simple $\mathrm{IA}_{3}+\mathbf{Y}_{0}$ term $\Gamma \vdash M: \theta$ there exists a fully productive tuple of $\llbracket \Gamma \vdash \theta \rrbracket$-automata for $\Gamma \vdash M: \theta$.

Proof. We use structural induction. Whenever our constructions fail to preserve productiveness, we simply appeal to Lemma 2 to obtain an equivalent productive automaton.

Thanks to the equalities below and the fact that productive $G$-automata compose well (see Remark 2 (i))

$$
\begin{aligned}
& (\Gamma \vdash \lambda x . M)=(\Gamma, x \vdash M) \quad(\Gamma \vdash M ; N)=(\Gamma \vdash M) \cdot(\Gamma \vdash N) \\
& (\Gamma \vdash ! M)_{j}=(\Gamma \vdash M)_{j}^{r} \quad(\Gamma \vdash M:=N)=\sum_{j=0}^{\max }\left((\Gamma \vdash N)_{j} \cdot(\Gamma \vdash M)_{j}^{w}\right) \\
& \left(\Gamma \vdash \text { if } M \text { then } N_{1} \text { else } N_{2}\right\rangle=\left(\Gamma \vdash M D_{0} \cdot\left(\Gamma \vdash N_{2}\right)+\right. \\
& \left(\sum_{j=1}^{\max }\left(\Gamma \vdash M D_{j}\right) \cdot\left(\Gamma \vdash N_{1}\right)\right.
\end{aligned}
$$

the corresponding cases are easy. We can simply appeal to the inductive hypothesis and construct the new automata by connecting suitable final states with suitable initial states with $\epsilon$-transitions. Note that this does not violate determinism as final states do not have outgoing transitions. Equivalently, one could "glue" final states with initial ones suitably. If one performs the constructions for reachable final states only, productiveness will be preserved. The remaining cases are treated as follows:

The case of $\Gamma \vdash$ new $X$ in $M: \beta$. Suppose $\beta=$ com and $\langle\mathcal{A}\rangle$ is fully productive for $\Gamma, X: \operatorname{var} \vdash M: \beta$. Let us construct $\max +1$ copies of $\mathcal{A}$ denoted by $\mathcal{A}^{0}, \cdots, \mathcal{A}^{\max }$. We will use superscripts to refer to their states. The unique automaton $\mathcal{B}$ in the fully productive tuple for $\Gamma \vdash$ new $X$ in $M$ will consist of all these copies, the idea being that the index of the copy corresponds to the interim value stored in the variable $X$. The new initial state will be $i^{0}$, i.e. the initial state of $\mathcal{A}^{0}$. The set of final states will be $H=\bigcup_{h=0}^{\max } F^{h}$, i.e. the set of final states from all the copies $\mathcal{A}^{h}$ of $\mathcal{A}$. We make the following changes to the copies of $\mathcal{A}$. Because $\mathcal{A}$ is productive, we can assume that each $\mathcal{A}$-transition using the P-moves read ${ }_{X}$ or write $(k)_{X}(0 \leq k \leq \max )$ originating from the distinguished copy of var is followed by transitions on $i_{X}^{1}, \cdots, i_{X}^{d}$ and $o k_{X}$ respectively, where 
line break removed

ditto

inlined

ditto

inlined $\left\{i^{1}, \cdots, i^{d}\right\} \subseteq\{0, \cdots, \max \}$. To construct $\mathcal{B}$ we redirect transitions in the various copies of $\mathcal{A}$ as shown below

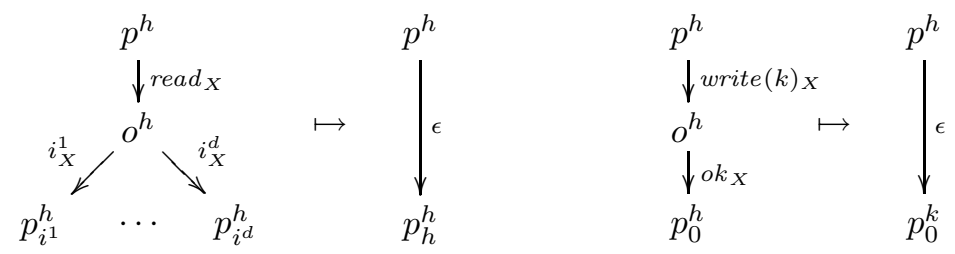

where $0 \leq h \leq \max$. These transformations redirect only the transition form $p^{h}$, while the transitions from $o^{h}$ remain as they were. In the first case the transformation is performed only if $h=i^{c}$ for some $1 \leq c \leq d$. Otherwise, the transition from $p^{h}$ is just erased. Note that in the second case the new transition connects $\mathcal{A}^{h}$ to $\mathcal{A}^{k}$. After all the transitions on P-moves are dealt with, we delete the transitions from $o^{h}$. It is clear that the $\mathcal{B}$ is careful if $\mathcal{A}$ is. Using Lemma 2 we can make it productive. If $\beta=\exp$ the same construction can be performed to give us a fully productive tuple for $\Gamma \vdash$ new $X$ in $M$. To define the required partition of states $H=\oplus_{j=0}^{\max } H_{j}$ we simply take $H_{j}=\bigcup_{h=0}^{\max } F_{j}^{h}$, where $F=\oplus_{j=0}^{\max } F_{j}$ is the partition given by the inductive hypothesis. The case of $\beta=\operatorname{var}$ combines the previous two cases.

The case of $\Gamma \vdash \mu x^{\beta} . M: \beta$. Suppose $\beta=\operatorname{com}$ and $\langle\mathcal{A}\rangle$ is a fully productive tuple for $\Gamma, x: \beta \vdash M: \beta$, where $\mathcal{A}=\langle Q, \Omega, i, F, \delta\rangle$. The unique automaton $\mathcal{B}$ in the fully productive tuple for $\Gamma \vdash \mu x . M$ will be constructed from two copies $\mathcal{A}^{0}, \mathcal{A}^{1}$ of $\mathcal{A}$. Intuitively, $\mathcal{A}^{1}$ will be used to process recursive calls from $M$, whereas $\mathcal{A}^{0}$ will correspond to the base copy of $M$. Accordingly, the initial state will be $i^{0}$ and the final states will be those from $F^{0}$. The stack alphabet of $\mathcal{B}$ will, in addition to the stack alphabet of $\mathcal{A}$, contain two copies of the set of O-states of $\mathcal{A}$. By productiveness of $\mathcal{A}$, we can assume that each transition on run $_{x}$ is followed by a transition on done $e_{x}$. In order to define $\mathcal{B}$, for each $h=0,1$ and for each block of the shape $p^{h} \stackrel{\text { run }_{x}}{\longrightarrow} o^{h} \stackrel{\text { done }_{x}}{\longrightarrow} p_{\text {done }}^{h}$ we erase the transition from $p^{h}$ and add the transitions: $p^{h} \stackrel{\epsilon / o^{h}}{\longrightarrow} i^{1}$ and $f^{1} \stackrel{\epsilon, o^{h}}{\longrightarrow} p_{\text {done }}^{h}$ for any $f \in F$. The case of $\beta=\exp$ is similar but, additionally, we have to pass on the result of the recursive call. The notion of a productive tuple will make that easy. Suppose $F=\oplus_{j=0}^{\max } F_{j}$. Then we first create two copies $\mathcal{A}^{0}, \mathcal{A}^{1}$ of $\mathcal{A}$ and for $h=0,1$ and for each block

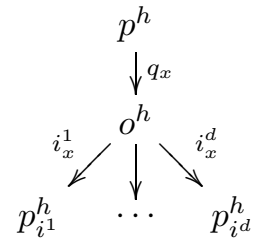

we erase the transition from $p^{h}$ and add the transitions: $p^{h} \stackrel{\epsilon / o^{h}}{\longrightarrow} i^{1}$ and $f_{i^{c}}^{1} \stackrel{\epsilon, o^{h}}{\longrightarrow}$ $p_{i^{c}}^{h}$ for any $1 \leq c \leq d$ and $f_{i^{c}} \in F_{i^{c}}$. The initial state of the new automaton is $i^{0}$, 
the final states are those in $F^{0}=\oplus_{j=0}^{\max } F_{j}^{0}$. The remaining case of $\beta=v a r$ is more tedious but completely analogous to the previous two. Given a fully productive tuple $\left\langle\mathcal{A}^{1}, \cdots, \mathcal{A}^{\max +2}\right\rangle$ for $\Gamma, x \vdash M$, blocks write $(j)_{x} o k_{x}$ are replaced with calls to $\mathcal{A}^{j+2}$ as for com, while blocks read $j_{x}$ are replaced by calls to $\mathcal{A}^{1}$. It is easy to see that the obtained automaton is careful. After the above constructions we can apply Lemma 2 to make the resulting automaton productive.

The case of $f M_{1} \cdots M_{k}$. For demonstration we assume that $k=1, M_{1}=M$ and $f M: c o m$ and $f$ does not occur in $M$, i.e. $f: \theta \rightarrow \operatorname{com}, \Gamma \vdash f M: c o m$. Let us

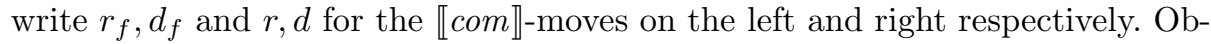
serve that because $f M$ is interpreted by interaction of the identity strategy (cor-

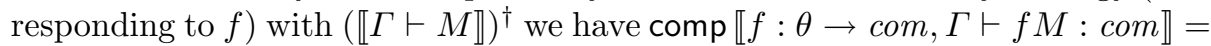
$r r_{f}(\{\epsilon\} \cup L) d_{f} d$, where $L=\phi\left(\operatorname{comp} \llbracket \Gamma \vdash M: \theta \rrbracket^{\dagger}\right)$ and $\phi$ is the (injective) renaming map which acts like identity on moves from $\llbracket \Gamma \rrbracket$ and maps $\llbracket \theta \rrbracket$-moves to their copies in $\llbracket \theta \rightarrow c o m \rrbracket$. Below we examine two representative examples in detail and give the precise shape of $\phi$. We use subscripts to refer to (moves from) various copies of com.

- Suppose $\Gamma \vdash M: \operatorname{com}_{0}$ and $f: \operatorname{com}_{f, 1} \rightarrow \operatorname{com}_{f}, \Gamma \vdash f M: c o m$. Then $L=$ comp $\llbracket \Gamma \vdash M \rrbracket^{\dagger}\left[r_{0} \mapsto r_{f, 1}, d_{0} \mapsto d_{f, 1}\right]$.

- Suppose $\Gamma \vdash M: \operatorname{com}_{1} \rightarrow \operatorname{com}_{0}$ and $f:\left(\operatorname{com}_{f, 2} \rightarrow \operatorname{com}_{f, 1}\right) \rightarrow \operatorname{com}_{f}, \Gamma \vdash$ fM : com. Then $L=$ comp $\llbracket \Gamma \vdash M \rrbracket^{\dagger}\left[r_{0} \mapsto r_{f, 1}, d_{0} \mapsto d_{f, 1}, r_{1} \mapsto r_{f, 2}, d_{1} \mapsto\right.$ $\left.d_{f, 2}\right]$.

Consequently, the main difficulty is the construction of an automaton accepting $\operatorname{comp}\left(\sigma^{\dagger}\right) \cup\{\epsilon\}$, where $\sigma=\llbracket \Gamma \vdash M: \theta \rrbracket$ and $\operatorname{ord}(\theta) \leq 1$. What is constructed in the following is not a $\llbracket \Gamma \vdash \theta \rrbracket$-automaton (the initial state, which is an O-state, will coincide with the final one and it will have both incoming and outgoing transitions), but the crucial fact is that it becomes one when the transitions on $r_{f}$ and $d_{f}$ (which will become the new initial and final moves respectively) are added. We consider the case of $\theta=\operatorname{com}_{1} \rightarrow \operatorname{com}_{0}$ for illustration.

Suppose $\langle\mathcal{A}\rangle$ is fully productive for $\Gamma \vdash M: \theta, \mathcal{A}=\langle Q, \Omega, i, F, \delta\rangle$ and $\operatorname{ord}(\theta) \leq 1$. Recall from Remark 1 that in this case $\dagger$ iterates as well as stacks copies of the original strategy and a new copy can start either if the previous one finished or after $r_{1}$. To model that, we create two copies $\mathcal{A}^{0}, \mathcal{A}^{1}$ of $\mathcal{A}$ with the aim of delegating the copies started after $r_{1}$ to $\mathcal{A}^{1}$. The outermost iterated copies will be processed by $\mathcal{A}^{0}$.

First we define a few new states: $i_{\text {new }}^{0}$ (which will be the initial and final state of the automaton) and $(\circ, q),(\bullet, q)$, where $q \in Q^{0}+Q^{1}+\left\{i_{\text {new }}^{0}\right\}$. We make the following additions to $\mathcal{A}^{0}, \mathcal{A}^{1}$ :

$-i_{\text {new }}^{0} \stackrel{r_{0}}{\longrightarrow}\left(\bullet, i_{\text {new }}^{0}\right) \stackrel{\epsilon /\left(i_{\text {new }}^{0}, r_{0}\right)}{\longrightarrow} i^{0}$ and $f^{0} \stackrel{\epsilon,\left(i_{\text {new }}^{0}, r_{0}\right)}{\longrightarrow}\left(\circ, i_{\text {new }}^{0}\right) \stackrel{d_{0}}{\longrightarrow} i_{\text {new }}^{0}$ for any $f \in F$.

- Observe that $r_{1}$ is always followed by $d_{1}$ in $\sigma$. By productiveness of $\mathcal{A}$, we can assume that each transition $p^{h} \stackrel{r_{1}}{\longrightarrow} o^{h}(h=0,1)$ is followed by $o^{h} \stackrel{d_{1}}{\longrightarrow} p_{0}^{h}$. Then we add $o^{h} \stackrel{r_{0}}{\longrightarrow}\left(\bullet, o^{h}\right) \stackrel{\epsilon /\left(o^{h}, r_{0}\right)}{\longrightarrow} i^{1}$. This makes it possible for the 
automaton to start processing a new copy of $\sigma$, the return state is saved on the stack. To process call returns, we add $f^{1} \stackrel{\epsilon,\left(o^{h}, r_{0}\right)}{\longrightarrow}\left(\circ, o^{h}\right) \stackrel{d_{0}}{\longrightarrow} o^{h}$ for any $f \in F$.

Note that in addition to return addresses in the form of O-states we have also arranged for the questions $r_{0}$ to be pushed on the stack. They will remain there as long as they are not answered in the corresponding position. These questions are redundant for accepting $\operatorname{comp}\left(\sigma^{\dagger}\right) \cup\{\epsilon\}$, but are necessary for the resulting automaton to be careful. Since $r_{0}$ will be substituted by $r_{f, 1}$, the questions $r_{0}$ are exactly the second-order questions contributed by $f$. The case of $f M_{1} \cdots M_{k}$ is analogous, except that we need to apply $\dagger$ to $\sum_{i=1}^{k} \llbracket \Gamma \vdash M_{i} \rrbracket$.

The contraction rule. The contraction rule for free identifiers of type $\theta$, where $\operatorname{ord}(\theta)=0,1$, is interpreted simply by identifying moves from the two copies of $\llbracket \theta \rrbracket$. In general this might lead to nondeterminism, but thanks to the structure of our automata this can never happen in our case. There is no problem with $\mathrm{P}$-moves as from each P-state the automaton can read at most one letter. Suppose that a nondeterminism arises from some O-state reachable from the initial configuration. This means that there exists a position $s$ such that the automaton can read both $s o_{1}$ and $s o_{2}$, where $o_{1}$ and $o_{2}$ are the O-moves from two different copies of $\theta$. However, this contradicts that fact that at most one of $s \mathrm{O}_{1}$ and $s \mathrm{O}_{2}$ can satisfy Visibility (because only one first-order question is O-visible in $s$ ). Consequently, contraction can be interpreted without loss of determinacy.

\section{Representing pointers}

We are going to introduce a representation of pointers for simple terms by following Lemma 1. Consider a position $s q_{3}$, where $q_{3}$ is a third-order question. We define $\pi\left(s, q_{3}\right)=k-j+2$ where $q_{2}^{j}$ is the last enabler of $q_{3}$ in the sequence $q_{2}^{1}, \cdots, q_{2}^{k}$ of all second-order unanswered questions in $s$ (written in the order they appear in $s$ ).

Definition 5. Suppose $\sigma=\llbracket \Gamma \vdash M: \theta \rrbracket$, where $\Gamma \vdash M: \theta$ is an $\mathrm{IA}_{3}+\mathbf{Y}_{0}$ term. The language $\mathcal{P}(\sigma)$ over the alphabet $M_{\llbracket \Gamma \vdash \theta \rrbracket}+\{$ check $\}$ is defined by $\mathcal{P}(\sigma)=\left\{\right.$ check $^{\pi\left(s, q_{3}\right)} \mid s q_{3} s^{\prime} \in \operatorname{comp} \sigma$ for some $\left.s^{\prime}\right\}$.

Lemma 3. For any simple term $\Gamma \vdash M: \theta$ there exists a DPDA accepting $\mathcal{L}(\operatorname{comp} \sigma) \cup \mathcal{P}(\sigma)$, where $\sigma=\llbracket \Gamma \vdash M: \theta \rrbracket$.

Proof. By Theorem 2 there is a fully productive tuple for $\Gamma \vdash M: \theta$. By adding transitions on the missing initial and final moves, we can construct an automaton $\mathcal{A}$ accepting $\mathcal{L}(\operatorname{comp} \sigma)$. Though $\mathcal{A}$ is not, strictly speaking, a $G$-automaton, it is nonetheless a DPDA that is productive and careful. (The definitions of productive and careful do carry over in this case.) Next, we will construct a DPDA accepting $\mathcal{L}(\operatorname{comp} \sigma) \cup \mathcal{P}(\sigma)$. Suppose that the automaton $\mathcal{A}$ reads the sequence $s q_{3}$ where $q_{3}$ is a third-order question. Note that $q_{3}$ is always a P-move, 
so it is uniquely determined by $s$ and $\sigma$. As $\mathcal{A}$ is productive, we know that $s q_{3}$ can be extended to a complete position. It remains to take care of check letters. As $\mathcal{A}$ is careful, all second-order questions that are open in $s q_{3}$ will be stored on the stack. Hence, in order to accept the right number of check's after reading $s$, the automaton can move to a fresh state (reading the letter check) and enter a new mode in which it will repeatedly pop the stack reading check whenever the topmost stack symbol contains a second-order question and $\epsilon$ otherwise. It accepts exactly after it encounters the first enabler of $q_{3}$.

Now that we can represent the semantics of simple terms with pointers, the semantics of other $\beta$-normal terms can be obtained by renaming, because in order to type such terms we only need to perform contraction at order 2 and $\lambda$-abstraction. The former is done by relabelling (in the same manner as at order 0 or 1 ; the previous argument that determinism is preserved remains valid), the latter amounts to identity in the games setting. Consequently, we get:

Theorem 3. For any $\mathrm{IA}_{3}+\mathbf{Y}_{0}$ term $\Gamma \vdash M: \theta$ in $\beta$-normal form there exists a $D P D A$ accepting $\mathcal{L}(\operatorname{comp} \sigma) \cup \mathcal{P}(\sigma)$, where $\sigma=\llbracket \Gamma \vdash M: \theta \rrbracket$. Hence, the theorem also holds for any $\mathrm{IA}_{3}+\mathbf{Y}_{0}$ term.

By Lemma $1, \mathcal{L}(\operatorname{comp} \sigma) \cup \mathcal{P}(\sigma)$ is a faithful representation of comp $\sigma$. Thus, by Theorem 3 and the decidability of DPDA equivalence [8], we get

Theorem 4. Observational equivalence is decidable for terms in $\mathrm{IA}_{3}+\mathbf{Y}_{0}$.

\section{Hardness}

A DPDA [6] is a tuple $\mathcal{B}=\left\langle Q, \Sigma, \Gamma, \delta, q_{0}, Z_{0}\right\rangle$, where $\delta: Q \times(\Sigma \cup\{\epsilon\}) \times \Gamma \rightarrow$ $Q \times \Gamma^{*}$. Additionally, whenever $\delta(q, a, X)$ is defined for some $a \in \Sigma, \delta(q, \epsilon, X)$ must be undefined. We consider acceptance by empty stack (initially the stack contains $Z_{0}$ ) and write $N(\mathcal{B})$ for the language accepted by $\mathcal{B}$. For simplicity, we further assume that $\mathcal{B}$ can either pop the stack or push one symbol onto it, i.e. if $\delta(q, a, X)=\left(q^{\prime}, \alpha\right)$ then $\alpha=\epsilon$ or $\alpha=\alpha_{0} \alpha_{1}\left(\alpha_{0}, \alpha_{1} \in \Gamma\right)$ and $\alpha_{0}=X$. Any DPDA can be easily converted into this form.

We identify values of type $\exp$ with $\Sigma$. Consider the game $G=\llbracket \exp \rrbracket \Rightarrow \llbracket c o m \rrbracket$ so that we have $M_{G}=\{q\} \cup \Sigma \cup\{r, d\}$. Given $L \subseteq \Sigma^{*}$ define $\widehat{L} \subseteq M_{G}^{*}$ by $\widehat{L}=\left\{r q x_{1} \cdots q x_{n} d \mid x_{1} \cdots x_{n} \in L\right\}$. Note that $\widehat{L_{1}}=\widehat{L_{2}}$ iff $L_{1}=L_{2}$.

Lemma 4. For any DPDA $\mathcal{B}$ there exists a term $x: \exp \vdash M_{\mathcal{B}}:$ com such that $\mathcal{L}\left(\operatorname{comp} \llbracket x: \exp \vdash M_{\mathcal{B}}: \operatorname{com} \rrbracket\right)=\widehat{N(\mathcal{B})}$.

Proof. Push transitions are simulated by recursive calls, pop moves by call returns. Before each call, the symbol to be pushed is stored in the variable TOP 
which is used to initialize the local copy of $X$ after the call. Take $M_{\mathcal{B}}$ to be

$$
\begin{aligned}
& x: \exp \vdash \text { new } Q:=q_{0}, T O P:=Z_{0}, C H \text { in } \\
& \mu z^{c o m} \text {. new } P O P:=0, X:=! T O P \text { in } \\
& \text { while }(\text { not } ! P O P) \text { do } \\
& \left(\text { if } \delta(! Q, \epsilon, ! X)=\left(q^{\prime}, \alpha\right)\right. \text { then } \\
& \left(Q:=q^{\prime} ;\right. \\
& \text { if } \left.\alpha=\epsilon \text { then } P O P:=1 \text { else }\left(\left(T O P:=\alpha_{1}\right) ; z\right)\right) \\
& \text { else } \quad(C H:=x ; \\
& \text { if } \delta(! Q, ! C H, ! X)=\left(q^{\prime}, \alpha\right) \text { then } \\
& \left(Q:=q^{\prime} ;\right. \\
& \text { if } \left.\alpha=\epsilon \text { then } P O P:=1 \text { else }\left(\left(T O P:=\alpha_{1}\right) ; z\right)\right) \\
& \text { else } \left.\left.\Omega_{\text {com }}\right)\right): \operatorname{com}
\end{aligned}
$$

Proposition 1. For any DPDAs $\mathcal{B}_{1}, \mathcal{B}_{2}$ we have $N\left(\mathcal{B}_{1}\right) \subseteq N\left(\mathcal{B}_{2}\right)$ iff $M_{\mathcal{B}_{1}} \sqsubseteq M_{\mathcal{B}_{2}}$.

Observe that the term used in Lemma 4 is from $I A_{1}+\mathbf{Y}_{0}$. Since the Containment Problem for DPDAs is undecidable [6] we have:

Corollary 1. Observational approximation is undecidable for $\mathrm{IA}_{1}+\mathbf{Y}_{0}$ terms; observational equivalence is at least as hard as DPDA Equivalence.

section header re- Our results complete the classification of decidable and undecidable fragments moved of IA. The exact complexity of observational equivalence for $\beta$-normal terms is known in most cases, as shown in the table in Section 1. The complexity of $\mathrm{IA}_{i}+\mathbf{Y}_{0}$ depends on the complexity of DPDA Equivalence, which is not known at present. Our construction, when suitably optimized, yields DPDAs that are doubly exponentially larger than the given term in $\beta$-normal form. It is also not

Figure 2. erased yet understood how the presence of $\beta$-redexes affects the complexity.

\section{References}

1. Ong, C.-H. L.: Observational equivalence of 3rd-order Idealized Algol is decidable. In: Proceedings of LICS (2002) 245-256

2. Murawski, A. S.: On program equivalence in languages with ground-type references. In: Proceedings of LICS (2003) 108-117

3. Murawski, A. S.: Games for complexity of second-order call-by-name programs. Theoretical Computer Science, to appear.

4. Murawski, A. S., Walukiewicz, I.: Third-order Idealized Algol with iteration is decidable. In: Proceedings of FOSSACS. LNCS 3441 (2005) 202-218

5. Stirling, C.: Deciding DPDA equivalence is primitive recursive. In: Proceedings of ICALP. LNCS 2380 (2002) 821-832

6. Hopcroft, J. E., Ullman, J. D.: Introduction to Automata Theory, Languages and Computation. Addison-Wesley (1979)

7. Abramsky, S., McCusker, G.: Linearity, sharing and state: a fully abstract game semantics for Idealized Algol with active expressions. In O'Hearn, P.W., Tennent, R.D., eds.: Algol-like languages, Birkhaüser (1997) 297-329

8. Sénizergues, G.: $\mathrm{L}(\mathrm{A})=\mathrm{L}(\mathrm{B})$ ? decidability results from complete formal systems Theoretical Computer Science 251(1-2) (2001) 1-166 